\title{
Ecological speciation and phenotypic plasticity affect ecosystems
}

\author{
Bänz Lundsgaard-Hansen, ${ }^{1,2,4}$ Blake Matthews, ${ }^{2,3}$ and Ole Seehausen ${ }^{1,2}$ \\ ${ }^{1}$ Division of Aquatic Ecology and Evolution, Institute of Ecology and Evolution, University of Bern, Baltzerstrasse 6, \\ CH-3012 Bern, Switzerland \\ ${ }^{2}$ Eawag Swiss Federal Institute of Aquatic Science and Technology, Department of Fish Ecology and Evolution, Centre of Ecology, \\ Evolution and Biogeochemistry, Seestrasse 79, CH-6047 Kastanienbaum, Switzerland \\ ${ }^{3}$ Eawag Swiss Federal Institute of Aquatic Science and Technology, Department of Aquatic Ecology, Centre of Ecology, \\ Evolution and Biogeochemistry, Seestrasse 79, CH-6047 Kastanienbaum, Switzerland
}

\begin{abstract}
Phenotypic differences among closely related populations and species can cause contrasting effects on ecosystems; however, it is unknown whether such effects result from genetic divergence, phenotypic plasticity, or both. To test this, we reared sympatric limnetic and benthic species of whitefish from a young adaptive radiation in a common garden, where the benthic species was raised on two distinct food types. We then used these fish in a mesocosm experiment to test for contrasting ecosystem effects of closely related species and of plastically induced differences within a species. We found that strong contrasting ecosystem effects resulted more frequently from genetic divergence, although they were not stronger overall than those resulting from phenotypic plasticity. Overall, our results provide evidence that genetically based differences among closely related species that evolved during a young adaptive radiation can affect ecosystems, and that phenotypic plasticity can modify the ecosystem effects of such species.
\end{abstract}

Key words: adaptive radiation; common gardening experiment; Coregonus; direct trophic effects; ecoevolutionary dynamics; ecological speciation; indirect effects.

\section{INTRODUCTION}

It is well established that adaptation to contrasting ecological conditions can contribute to phenotypic diversification and speciation (Schluter 2000, Nosil 2012), but less is known about how phenotypic diversification, speciation, and adaptive radiation can affect ecosystem properties and functions (Harmon et al. 2009, Seehausen 2009, Schoener 2011). Recent experimental work has revealed that organisms from closely related populations with different phenotypes can have contrasting effects on a wide range of structural or functional aspects of ecosystems (Palkovacs and Post 2009, Bassar et al. 2010). For example, phenotypic differentiation in the foraging traits of allopatric alewife fish populations (Alosa pseudoharengus) affects the species composition, size structure, and life-history traits of their zooplankton prey (Post et al. 2008, Walsh and Post 2011). Similarly, a recent speciation event in stickleback (Gasterosteus aculeatus species complex), which is associated with adaptation to divergent ecological conditions in lakes (Bentzen and McPhail 1984, Schluter 1995, Rundle et al. 2000), affects prey community structure, as well as primary production and dissolved organic compounds in experimental aquatic mesocosms (Harmon et al. 2009). Such studies suggest

Manuscript received 18 December 2013; revised 19 March 2014; accepted 27 March 2014. Corresponding Editor: M. C. Urban.

${ }^{4}$ E-mail: baenz.lundsgaard@eawag.ch that the effects of evolutionary diversification can have far-reaching effects on ecosystems, but because these previous experiments only used wild-caught organisms, they cannot tell us whether the contrasting ecosystem effects of closely related populations or species are caused by genetic divergence or by environmentally induced differences (phenotypic plasticity).

Phenotypic plasticity is present when a single genotype can produce multiple forms of morphology, physiological state, and/or behavior in response to environmental differences (West-Eberhard 1989, 2003). Plasticity itself is an evolving trait with a genetic basis (Pigliucci 2005), and it is a common feature in adaptive radiations (i.e., Grant 1986, Day and McPhail 1996, Losos et al. 2000, Bouton et al. 2002, Robinson and Parsons 2002, West-Eberhard 2003, Wund et al. 2008). Plasticity may affect many ecological processes, such as population dynamics, trophic cascades, and species interaction networks (i.e., Raimondi et al. 2000, Agrawal 2001, Miner et al. 2005). For example, previous experiments have found that the composition of an intertidal rock community depends on whether or not barnacles display a predator-induced defense trait (Raimondi et al. 2000). Phenotypic plasticity could also explain the contrasting ecosystem effects of different populations of the same species (Post et al. 2008, Bassar et al. 2010, Matthews et al. 2011a) and of closely related species (Harmon et al. 2009), but this has not been tested. It is important to distinguish between ecosystem effects caused by genetic divergence and those caused by 

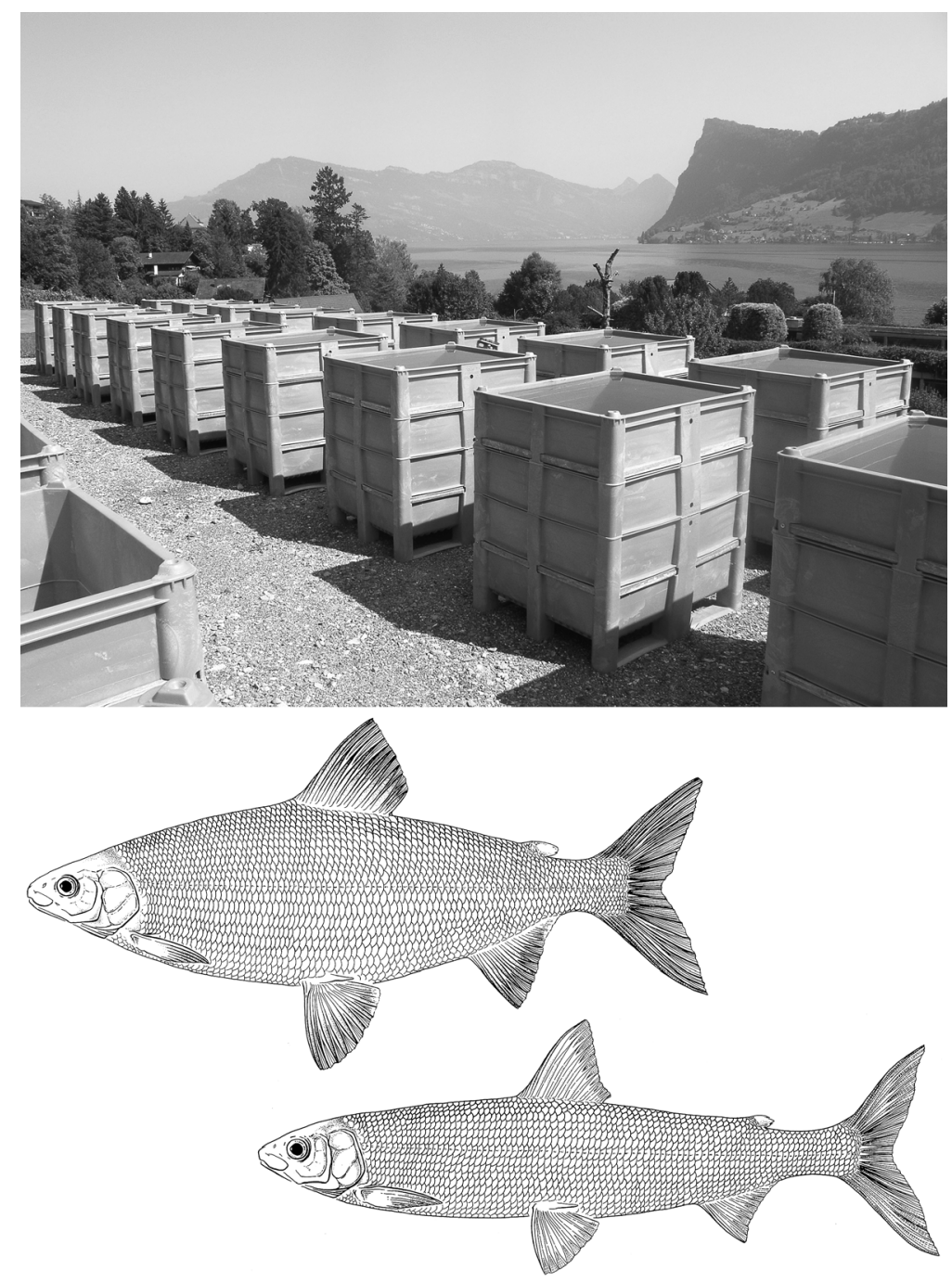

Plate 1. (Top) The mesocosm garden and (center) Coregonus sp. "Bodenbalchen" and (bottom) C. zugensis, the studied fish species. Photo credit: B. Lundsgaard-Hansen. Drawings by Verena Kaelin (Zurich).

environmentally induced phenotypes for a number of reasons. Genetic divergence is relatively slow to emerge, as it requires several generations, and it can be lost quickly when the gene-flow-selection-balance changes, whereas environmental induction happens within a single generation and is robust to changes in gene flow. If divergent ecosystem effects of sympatric species result primarily from environmentally induced (plastic) phenotypic differences among individuals, then evolutionary divergence of species may not be required for there to be intrapopulation or interspecific variation in organism-mediated ecosystem effects within a community (Seehausen 2009, Hanski 2012).

In this study, we used a sympatric benthic and limnetic species pair of whitefish to investigate whether genetic differentiation among closely related species and/or environmentally induced differentiation within species cause differential effects on aquatic ecosystems in mesocosms (hereafter referred to as ecosystems). We first raised whitefish in a common garden for three years (common garden refers to raising organisms in the same environments in order to quantify genetic differences), and we raised juveniles of one species on two distinct food types. We then used the reared fish as treatments in a subsequent common gardening experiment. In a common gardening experiment (sensu Matthews et al. 2011b) where the treatments are organisms with phenotypic (heritable or environmentally induced) differences among them, the goal is to quantify how they differ in their effects on ecosystem properties and functions (Matthews et al. 2011b). Our plasticity treatment focused on foraging traits because they are commonly implicated in ecosystem effects of predators (Palkovacs and Post 2009) and because they readily diverge during adaptive radiations, including the radiations of whitefish in pre-alpine lakes (Schluter 
2000, Lundsgaard-Hansen et al. 2013, Roesch et al. 2013).

In the adaptive radiations of Swiss whitefish, more than 30 species have arisen within less than 15000 years after the retreat of the Alpine ice sheets (Vonlanthen et al. 2012). Phylogenetic analyses suggest that this diversity of sympatric species has originated through at least five independent adaptive radiations that occurred in parallel in different lakes or lake systems (Hudson et al. 2011). The species we used here (Coregonus sp. "Bodenbalchen" and C. zugensis; see Plate 1) belong to the monophyletic radiation endemic to Lake Lucerne, Switzerland (Hudson et al. 2011). The benthic species $C$. sp. "Bodenbalchen" is larger at maturity and has fewer gill rakers than the limnetic species $C$. zugensis (Vonlanthen et al. 2012). This divergence in morphology is supported by experimental work showing strong heritable differences in these traits and also in feeding efficiencies between $C$. sp. "Bodenbalchen" and $C$. zugensis (Lundsgaard-Hansen et al. 2013, Roesch et al. 2013). However, phenotypic differentiation between these species is not limited to foraging traits, as they also differ in spawning depth, habitat use, and other traits (Woods et al. 2009, Karvonen et al. 2012, Vonlanthen et al. 2012).

We performed a common gardening experiment with the following three treatments: (1) the benthic species (B), C. sp. "Bodenbalchen," raised on benthic food (benthic benthic, BB), (2) the same benthic species raised on limnetic food (benthic limnetic, BL), and (3) the limnetic species (L), C. zugensis, raised on limnetic food (limnetic limnetic, LL). We refer to genetic effects on ecosystems when comparing treatments with fish belonging to different species but raised on the same food (BL vs. LL), to plasticity effects when comparing treatments with the same species raised on different food (BB vs. BL), and to combined effects when comparing treatments with different species raised on food sources matching their natural habitat (BB vs. LL). We did not use a full factorial design, and so the inference we make about the effects of phenotypic plasticity (BB vs. BL) pertain to the species we raised on both food types ( $C$. sp. "Bodenbalchen") and the inference we make about genetic effects pertain to fish raised on limnetic food (zooplankton). In the scenario that phenotypic plasticity solely determines the ecosystem effects of whitefish (Fig. 1a), we would expect mesocosms containing the same species raised on different food to generate contrasting ecosystem states, and mesocosms containing different species raised on the same food to have similar ecosystem states (see Table 1 for the suite of ecosystem metrics defining the ecosystem state). If genetic differences among species cause ecosystem effects that are independent of phenotypic plasticity, we would expect large contrasts between LL and BL and small contrasts between BL and BB (Fig. 1b). If ecosystem effects result from an additive combination of both adaptive plasticity and genetic differences, then we would expect mesocosms with the $\mathrm{BB}$ and the LL treatment to have the most divergent ecosystems, and those containing the BL treatment to be intermediate (Fig. 1c).

In a previous study of the effects of the rearing treatments (food) on the feeding efficiency of whitefish (Lundsgaard-Hansen et al. 2013), we found that both genetic differences and rearing environment had significant effects on foraging efficiency when fish fed on benthic food. Here, we use a subset of the same individuals for the mesocosm experiment in order to test four predictions about ecosystem effects of genetic differences and phenotypic plasticity. First, we predicted that ecosystem differences among treatments would arise due to both genetic divergence and environmentally induced differences. This is because we had previously found that behavioral differences in foraging efficiency on benthic food were partly heritable and partly due to different foraging environments during the rearing of the fish (plasticity; Lundsgaard-Hansen et al. 2013). Second, we predicted particularly strong effects of those phenotypic differences that arise from genetic differentiation. This is because some whitefish foraging traits are highly heritable (Bernatchez 2004), and also because species differences are not restricted to foraging traits, but also include other traits (e.g., shape, behavior, life history, and metabolism) that could contribute to contrasts in ecosystem effects (Vonlanthen et al. 2009, Woods et al. 2009, Karvonen et al. 2012, Blank et al. 2013). Third, we predicted that effects would be maximized when plasticity and genetic differences are combined (Fig. 1d). This is based on the observation of additive effects of genetic differences and plasticity on foraging behavior (LundsgaardHansen et al. 2013). Fourth, we predicted that contrasts between treatments would be larger for the direct consumptive effects of whitefish on their prey (referred to as trophic effects) rather than those resulting either from direct non-trophic effects (e.g., nutrient cycling) or from indirect trophic effects (e.g., cascading interactions; Fig. 1e). For simplicity, we collectively refer to such effects as non-trophic effects, even though some effects may be indirectly caused by trophic interactions. Predicting the relative size of trophic and non-trophic effects is not trivial in reticulate food webs where consumers and predators connect multiple food chains (Teng and McCann 2004). In aquatic ecosystems, predator-mediated cascading effects on lower trophic levels are relatively common (Borer et al. 2005, Baum and Worm 2009), and previous studies suggest that such effects might dampen further down the food chain (McQueen et al. 1989, Micheli 1999, Shurin et al. 2002). In addition, there is some evidence that genetic and ecological effects attenuate across different levels of organization, for example, from community to ecosystem properties (Bailey et al. 2009, Dickie et al. 2012). However, such attenuation is not always observed, and the distribu- 
tion of ecosystem effect sizes may depend on which traits underlie the ecosystem effects (Bassar et al. 2010, Palkovacs et al. 2012).

\section{Material And Methods \\ Common garden experiment}

Experimental fish were bred in the winter of 2006, using five females and five males of each species. Eggs and sperm were stripped in the lab and eggs of all five females of each species were mixed. The eggs were fertilized simultaneously with sperm from the five conspecific males, resulting in up to 25 half-sib families per species. Fish were raised for approximately three years in the lab and all juvenile fish were fed with zooplankton in the first year. After about one year, the juveniles of the benthic species were split into two different food treatments and were raised in these treatments for approximately two additional years. Frozen mosquito larvae (Chironomus plumosus) were used as benthic food, and zooplankton, collected from Lake Lucerne, Switzerland, five times a week, were used as limnetic food. This resulted in three different treatments: genetically benthic fish raised on benthic food (BB), genetically benthic fish raised on limnetic food (BL), and genetically limnetic fish raised on limnetic food (LL). Each treatment was distributed over two aquaria, each with a volume of $120 \times 71 \times 50 \mathrm{~cm}$. Initially, each aquarium contained 100 individuals. Over the entire rearing time, mortalities in the different aquaria were as follows: $\mathrm{BB}_{1}, 6 \% ; \mathrm{BB}_{2}, 7 \% ; \mathrm{BL}_{1}, 6 \%$; $\mathrm{BL}_{2}, 20 \% ; \mathrm{LL}_{1}, 2 \% ; \mathrm{LL}_{2}, 9 \%$. When densities of fish slightly diverged through time between rearing aquaria, food provisioning was adjusted and fish from the same treatment raised in different aquaria did not differ in their standard length at the end of the rearing time (Lundsgaard-Hansen et al. 2013). Additional details about the rearing of the fish can be found elsewhere (Lundsgaard-Hansen et al. 2013).

\section{Common gardening experiment}

In September 2009, we set up 20 experimental mesocosms $(1000 \mathrm{~L})$ to serve as replicated mesocosm ecosystems (see Plate 1). Mesocosms were filled with gravel, sand, and water from Lake Lucerne, and $2.46 \mathrm{~g}$ of $\mathrm{NaNO}_{3}$ and $0.18 \mathrm{~g} \mathrm{NaH}_{2} \mathrm{PO}_{4}$ (phosphorous and nitrogen) were added to each mesocosm to stimulate primary production. In order to achieve a high diversity of pelagic and benthic organisms, each tank was inoculated with a mix of sediments from three different nearby lakes (Sempach, Roth, and Lucerne), and additionally supplemented with zooplankton from Lake Lucerne at the beginning of the experiment. The inoculation was done in a way that ensured that starting conditions were similar in all tanks.

\section{Distribution of fish}

Fish were put into the mesocosms on 9 October 2009. Fish from the $\mathrm{BB}$ and the $\mathrm{BL}$ treatments were assigned to seven mesocosms each, fish from the LL treatment to six (in total 20 mesocosms). Fish biomass per mesocosm ranged from $51 \mathrm{~g}$ to $74 \mathrm{~g}$ per tank and was not significantly different among treatments (ANOVA: $P=$ $0.95)$. The number of fish per mesocosm varied between two and five with a total of 56 fish distributed over the 20 tanks. The number of fish was adjusted to maintain the same average biomass across treatments. Including fish number as a co-variable of treatment did not change the significance level of the treatment effect for any ecosystem metric we measured at the end of the experiment, and so we did not include fish number in further analyses. Fish behavior and health were checked daily by visual observation. Over the entire experiment, two fish died (mortality rate $=0.06$ ) and one developed an eye infection. These fish were removed immediately and replaced with an individual of similar weight from the same rearing treatment. After nine weeks the experiment was stopped and fish were removed and killed with an overdose of the anesthetic MS222 (Acros Organics, Geel, Belgium).

\section{Measuring ecosystem variables}

We measured the following ecosystem metrics: The density (D) and size (S) of macro- (MAZP) and micro- (MIZP) zooplankton (MAZPD, MAZPS, MIZPD, MIZPS), community composition of macrozooplankton (ZPC), the abundance of snails (SNA) and Dreissena mussels (DRA), chlorophyll $a$ as a proxy for phytoplankton biomass (PPC), benthic algae cover (BAC), sedimentation rate (SED), dissolved organic compounds (DOC), and light transmission of photosynthetically active radiation (PAR; see Appendix A for measuring dates). Macrozooplankton included species that whitefish are expected to forage on directly (observed mean size range per taxon and tank: 0.4-2 $\mathrm{mm}$ ), including acarids, Bosmina, calanoid copepods, cyclopoid copepods, and Daphnia; whereas microzooplankton (observed mean size range per taxon and tank: $0.1-0.3 \mathrm{~mm}$ ) included copepod nauplii and Rotifera. Ecosystem metrics were divided into trophic and nontrophic metrics (see Table 1). Details on how the different ecosystem metrics were measured can be found in Appendix B.

\section{Whitefish phenotypic differences}

To quantify phenotypic differences between our treatments, we measured the standard length of each fish before fish were put into the mesocosms and we counted the number of gill rakers after the removal of the fish from the mesocosms. We chose these traits because the number of gill rakers and fish standard length are among the traits that most often diverge between sympatric whitefish species in the course of adaptive radiation (i.e., Lu and Bernatchez 1999, Vonlanthen et al. 2012). Gill raker numbers and standard length were averaged for each tank and treatment differences were calculated at the 
a)

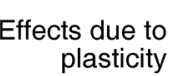

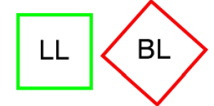

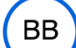

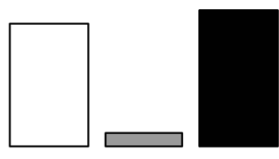

b) Effects due to genetic divergence
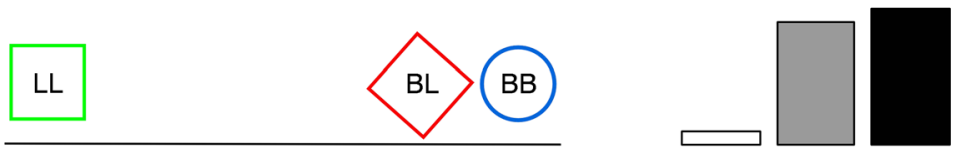

c) Combined effects of (a) and (b)
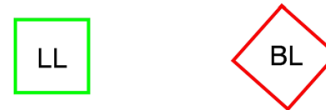

$$
\text { . }
$$

d) Predicted effect sizes
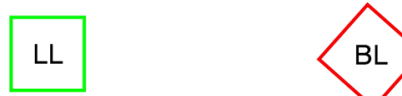

Ecosystem state

e) Predicted effect size distribution

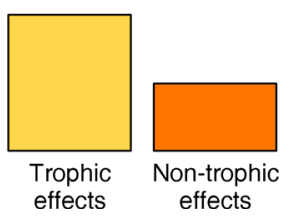

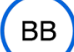
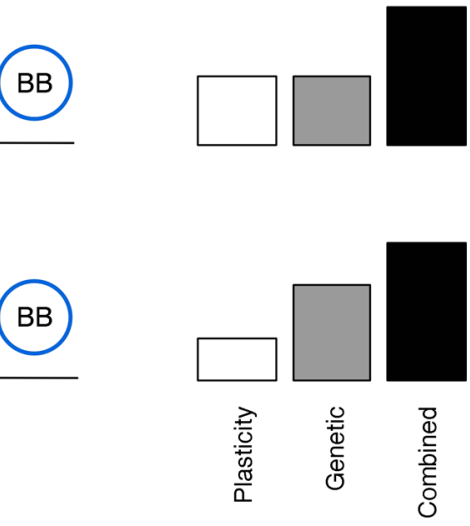

Effect size

FIG. 1. A conceptual figure of the study. Shown are expected ecosystem states (either averaged across all measured metrics or based on multivariate statistics) when ecosystem effects are a result of (a) phenotypic plasticity, (b) genetic species divergence, or (c) a combination of both. On the right side of each panel are associated effect sizes for the different contrasts; benthic species raised on benthic (BB) vs. limnetic (BL) food contrasts plasticity effects, BL species vs. limnetic species raised on limnetic food (LL) contrasts genetic effects, and BB vs. LL contrasts the combined effects. Bars are color coded and ordered as in subsequent figures (white represents plasticity, gray represents genetic, and black represents combined effects). Predictions are shown in panel (d). We predicted ecosystem effects to result from a combination of genetic species divergence and phenotypic plasticity, with overall greatest differences between $\mathrm{BB}$ and LL and stronger genetic than plasticity effects (treatment colors and symbols are as in subsequent figures, green squares represent LL, red diamonds represent BL, and blue circles represent BB). Further, we predicted that effects on trophic metrics (yellow bars) are stronger than effects on non-trophic (orange bars) metrics across all contrasts (panel e).

replication level of the mesocosm $(N=20)$ using KruskalWallis ANOVA and post-hoc Wilcoxon rank sum tests.

In an earlier study (Lundsgaard-Hansen et al. 2013), we measured body shape and several metrics of foraging behavior for the same fish we used in the current study, and found significant genetic effects on fish body shape and foraging behavior and significant plasticity effects on foraging behavior. For logistical reasons, we could not keep track of individual-level traits for those fish used in both the feeding trials and the mesocosm experiments. However, since all the fish used in the mesocosm experiment were also included in the previous foraging experiments (Lundsgaard-Hansen et al. 2013), we could randomly sample individuals from the previous study in order to estimate expected phenotypic differences among treatments in our mesocosm experiment. We did 1000 random sampling trials and used Wilcoxon rank sum tests to determine whether we would expect significant phenotypic differences for the plasticity and the genetic contrast in the mesocosm experiment. For the genetic contrast, the average $P$ values are 0.24 for body shape (Fig. 2a), 0.02 for latency time (Fig. 2b), 0.01 for foraging time (Fig. 2c), and 0.22 for the number of unsuccessful attacks (Fig. 2d; for more details about the measured metrics see Lundsgaard-Hansen et al. [2013]). For the plasticity contrast, the average $P$ values are 0.67 for body shape, 0.11 for foraging time, 0.35 for the number of unsuccessful attacks, and 0.54 for latency time.

\section{Statistical analysis}

We used principal component analysis (PCA) and Kruskal-Wallis ANOVA to test for multivariate diver- 
TABLE 1. The ecosystem metrics and their response to differences among whitefish.

\begin{tabular}{|c|c|c|c|c|c|c|}
\hline Metric & Acronym & Type & Times sampled & Flatness test & Levels test & Parallelism test \\
\hline Snail abundance (no. $\left./ \mathrm{m}^{2}\right)$ & SNA & trophic & 4 & ns & 0.003 & ns \\
\hline Dreissena abundance (no. $\left./ \mathrm{m}^{2}\right)$ & DRA & trophic & 4 & ns & 0.036 & ns \\
\hline Macrozooplankton density (no./L) & MAZPD & trophic & 3 & 0.02 & 0.031 & ns \\
\hline Macrozooplankton size (mm) & MAZPS & trophic & 3 & 0.04 & ns & ns \\
\hline Macrozooplankton community & $\mathrm{ZPC}$ & trophic & 3 & & & \\
\hline Microzooplankton density (no./L) & MIZPD & non-trophic & 3 & ns & ns & ns \\
\hline Microzooplankton size (mm) & MIZPS & non-trophic & 3 & ns & ns & ns \\
\hline Phytoplankton concentration $(\mathrm{mg} / \mathrm{L})$ & PPC & non-trophic & 7 & $<0.001$ & ns & 0.046 \\
\hline Dissolved organic compounds $(\mathrm{mg} / \mathrm{L})$ & DOC & non-trophic & 5 & 0.024 & ns & ns \\
\hline Photosynthetically active radiation (\%) & PAR & non-trophic & 3 & 0.009 & ns & ns \\
\hline Benthic algae cover (\%) & BAC & non-trophic & 3 & ns & ns & ns \\
\hline Sedimentation rate $(\mathrm{mg} / \mathrm{d})$ & SED & non-trophic & 1 & & & \\
\hline
\end{tabular}

Notes: Given are the measured ecosystem metrics, their acronym, the type of the metric, the number of times they were sampled, and their significance levels in the three tests implemented in profile analysis (see Materials and methods). Variables that are affected by direct trophic effects of differences between whitefish are called trophic variables, while variables which are not directly affected by trophic interaction of whitefish with their prey are called non-trophic variables. Metrics with less than three repeated measures and their acronyms are listed, but no profile analysis (PA) could be done for these (cells left blank). Redundancy analysis, which we used to estimate zooplankton community composition (ZPC) was calculated separately for the three measuring dates; values are thus not comparable across time and PA could therefore not be done on ZPC. Nonsignificant tests $(P>0.05)$ are indicated by "ns".

gence in ecosystem state among treatments (more details in Appendix C). We used profile analysis (PA) on logtransformed response variables to evaluate whether fish from different treatments affected temporal dynamics of ecosystems differently (see Table 1). Profile analysis is an alternative to repeated measure ANOVA (RMA), but the assumptions are less restrictive (Tabachnik and Fidell 2006). PA includes three different tests: (1) a flatness test, which tests whether profiles fluctuate or whether they are flat over time (similar to a time effect in RMA), (2) a levels test, which tests whether treatments differ in their average levels over time (similar to a treatment effect in RMA), and (3) a parallelism test, which tests whether different profiles are parallel over time (similar to a time/treatment interaction in RMA).
We used linear modeling and backward model selection based on a corrected Aikake information criterion, $\mathrm{AIC}_{\mathrm{c}}$ (Appendix F; Burnham and Anderson 1998), to investigate factors influencing phytoplankton abundance across all mesocosms. We used redundancy analysis (RDA; Legendre and Gallagher 2001) to assess differences in macrozooplankton community composition (ZPC) between treatments. We used the absolute value of Cohen's $d$ (Cohen 1988) to calculate effect sizes for each contrast (plasticity, genetic, combined) and we tested for significance using randomizations (Bailey et al. 2009). Cohen's $d$ is the difference in means of two treatments corrected for their pooled standard deviation (Cohen 1988). We used Kruskal-Wallis ANOVA (KWA) and post-hoc Mann Whitney tests to analyze

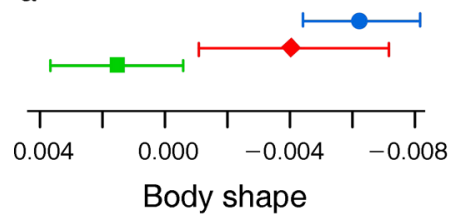

d

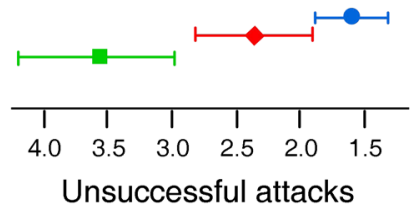

b

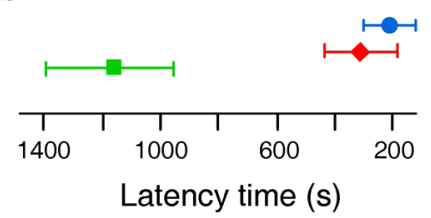

e

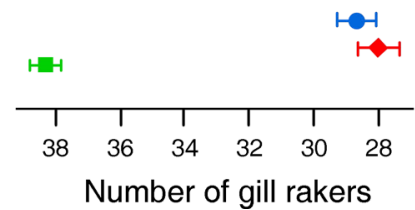

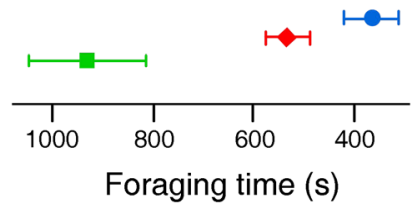

f

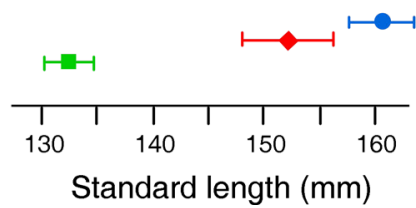

FIG. 2. Phenotypic and behavioral differences (with standard deviation) between treatments. Treatment colors and symbols are as in Fig. 1. Panels (a-d) show phenotypic and behavioral differences measured in a previous study using the same individuals as the current study (Lundsgaard-Hansen et al. 2013). Values in panel (a) correspond to principal component (PC) scores from a geometric morphometrics analysis, and a low value corresponds to a more downturned head. In panels (b-d), a low value corresponds to increased feeding efficiency on benthic food (more details in Lundsgaaard-Hansen et al. 2013). The (e) number of gill rakers and (f) fish standard length were measured during the current study. Overall, genetically based differences between treatments are stronger than plasticity differences between rearing environment. Nevertheless, the BL treatment is intermediate to the $\mathrm{BB}$ and the LL treatment in all foraging traits and behaviors, except in the number of gill rakers. 
differences among treatments in ecosystem metrics for each sampling date (see Appendix D).

All statistical analyses were performed using R ( R Development Core Team 2010).

\section{RESUlts}

Phenotypic differences of whitefish among treatments

Fish from different treatments (LL, BL, and BB) were significantly different in the morphological traits we measured at the end of the experiment, including gill raker numbers and standard length (Fig. 2e, f). Post-hoc tests revealed that differences resulted from genetic divergence and not from phenotypic plasticity (Fig. $2 \mathrm{e}, \mathrm{f})$. Also, when we reanalyzed the body shape and behavioral trait data that we collected in a previous study using the same individuals (Lundsgaard-Hansen et al. 2013) at the replication level of the mesocosm, we did not find significant effects of rearing treatment on morphology or behavior (Fig. 2a-d, for more statistical details see Materials and methods). Overall, phenotypic and behavioral differences between treatments were stronger for the genetic contrast than for the plasticity contrast. Inspection of phenotypic differences suggests that observed effects of plasticity on the ecosystem may be mediated through either an interaction of multiple weak phenotypic differences between our plasticity treatments or plasticity in unmeasured traits (Fig. 2a-d).

\section{Multivariate analysis of ecosystem divergence}

At the end of the experiment, multivariate analysis of ecosystem state revealed significant differences between treatments (Appendices $\mathrm{C}$ and $\mathrm{D}$ ), with divergence along the first principle component axis being greatest between $\mathrm{BB}$ and $\mathrm{LL}$ treatments, and the BL treatment being intermediate (Fig. 1d, Fig. 3a, Appendix D). This was consistent with our predictions that differences among treatments would arise due to both genetic divergence and environmentally induced differences, but that effects would be maximized when plasticity and genetic differences are combined. Along the second axis, the three treatments were also significantly different, and divergence was greatest between BL and LL (Fig. 3a, Appendix D), consistent with our prediction of strong effects of phenotypic differences that arise from genetic differentiation. When only trophic metrics were included in this analysis, the treatments differed significantly with respect to the first $\mathrm{PC}$ axis, again with greatest differences between BB and LL (Fig. 3b, Appendix D), but not the second axis (Fig. 3b, Appendix D). By comparison, when only non-trophic metrics were included, there were no differences between treatments on any axis (Fig. 3c, Appendix D), consistent with our prediction that contrasts between treatments would be larger for the direct trophic than for non-trophic effects.

\section{Direct trophic effects of whitefish}

The majority of metrics associated with the prey community (trophic metrics) differed significantly
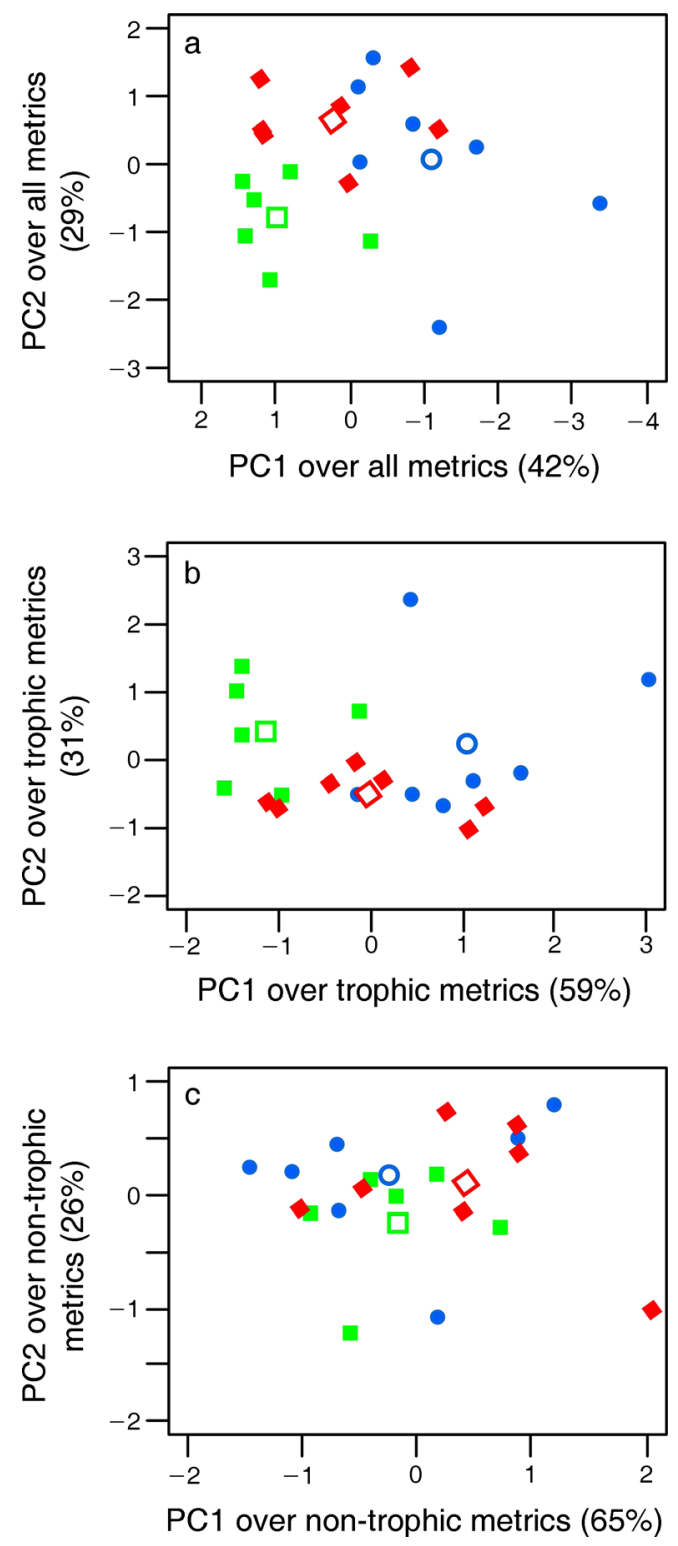

FIG. 3. Multivariate ecosystem divergence at the end of the experiment. Shown are PC scores of each tank (filled symbols; colors as in Fig. 1) and treatment average scores (open symbols) for (a) PC1 (42\%) and PC2 (29\%) across all metrics, (b) PC1 $(59 \%)$ and PC2 (31\%) across all trophic variables, and (c) PC1 $(65 \%)$ and PC2 (26\%) across all non-trophic variables. The loadings of the different metrics on the different axis are given in Appendix C, statistical tests on PC scores are reported in Appendix D.

among whitefish treatments in their average values over the entire duration of the experiment (see the significant level tests in PA of Table 1, Fig. 4). As examples, the average abundance of snails, Dreissena mussels, and macrozooplankton were all significantly affected by the whitefish treatments, albeit in different ways. Snail and Dreissena abundance was lowest in the BB treatment, (Fig. 4a, b), and macrozooplankton density was lowest 

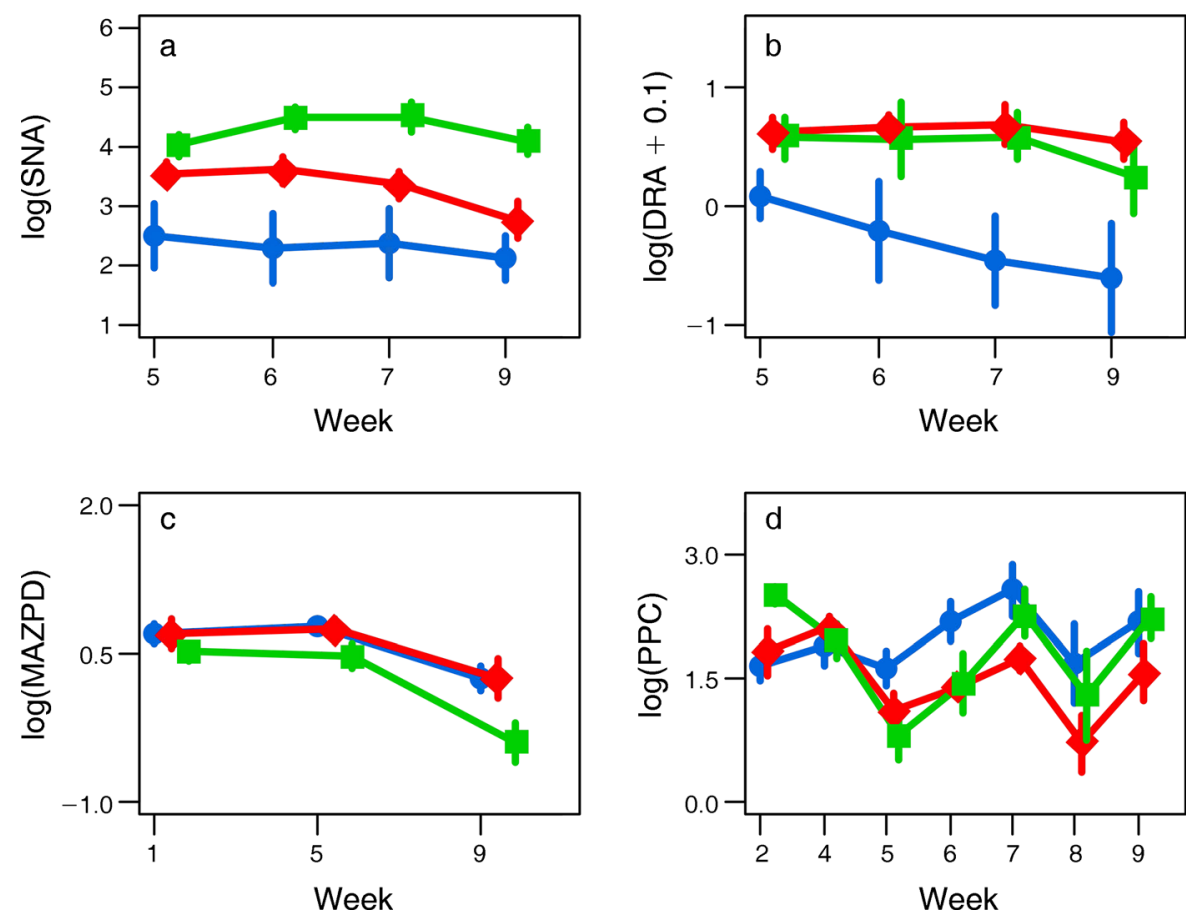

FIG. 4. Analysis of temporal variation of ecosystem effects. Shown is the time (weeks) after start of the common gardening experiment on the $x$-axis and different ecosystem parameters on the $y$-axis. Acronyms of the different panels are as in Table 1 and colors are as in Fig. 1. Only metrics with either significant levels or parallelism tests in profile analysis (PA) are shown (see Table 1): (a) snail abundance (SNA), (b) Dreissena abundance (DRA), (c) macrozooplankton density (MAZPD), and (d) phytoplankton concentration (PPC).

in the LL treatment (Fig. 4c). In addition, the different whitefish treatments had contrasting effects on the community composition of zooplankton (ZPC) at the end of the experiment (Fig. 5; Appendices D and E).

\section{Non-trophic effects of whitefish}

The temporal dynamics of phytoplankton were significantly different among the three treatments. This is an example of indirect effects of whitefish divergence on ecosystems (see the significant parallelism test from PA in Table 1, Fig. 4d). Such non-parallelism in the phytoplankton response between treatments probably reflects variation over the duration of the experiment in the factors regulating phytoplankton biomass. Indeed, phytoplankton concentration was negatively associated with the size of macrozooplankton in the middle of the experiment $(P=0.046$, Appendix $F)$ but was significantly negatively associated with Dreissena abundance at the end of the experiment $(P=0.04$, Appendix F).

\section{Distribution of effect sizes among contrasts}

Consistent with the first three predictions, the average ecosystem effect size (absolute Cohen's $d$ ) at the end of the experiment was 0.48 for the plasticity contrast (minimum $=0.001$, maximum $=1.38), 0.83$ for the genetic contrast (minimum $=0.05$, maximum $=2.41$ ), and 0.91 for the combined contrast (minimum $=0.07$, maximum $=2.95$; Fig. 5), but these differences were nonsignificant (all paired $t$ tests nonsignificant). Nevertheless, across all metrics (see Table 1), only one (Dreissena abundance) out of 12 differed significantly between the plasticity contrasts (based on randomization tests of Cohen's $d$; Bailey et al. 2009), while for both the genetic and combined effects, the number of significant tests was much higher (five out of 12 each, Fig. 5). The lower number of significant contrasts resulting from the plasticity treatment was also evident when looking at the density and size variation of individual zooplankton taxa (Fig. 6). In this case, plasticity contrasts were never significant, but the genetic contrasts were significantly different in six out of 10 cases, and the combined contrasts in five out of 10 cases (Fig. 6).

\section{DISCUSSION}

The ecological mechanisms and evolutionary pathways of ecological speciation and adaptive radiation have received considerable attention (Schluter 2000, Nosil 2012), but much less is known about their ecosystem consequences. Importantly, species that evolve in adaptive radiations are often shaped by ecological interactions in sympatry. Such species are expected to be functionally different more often than other closely related species. Does it make a difference to the functioning of ecosystems whether ecosystems host a diverse radiation of organisms all closely related to each 


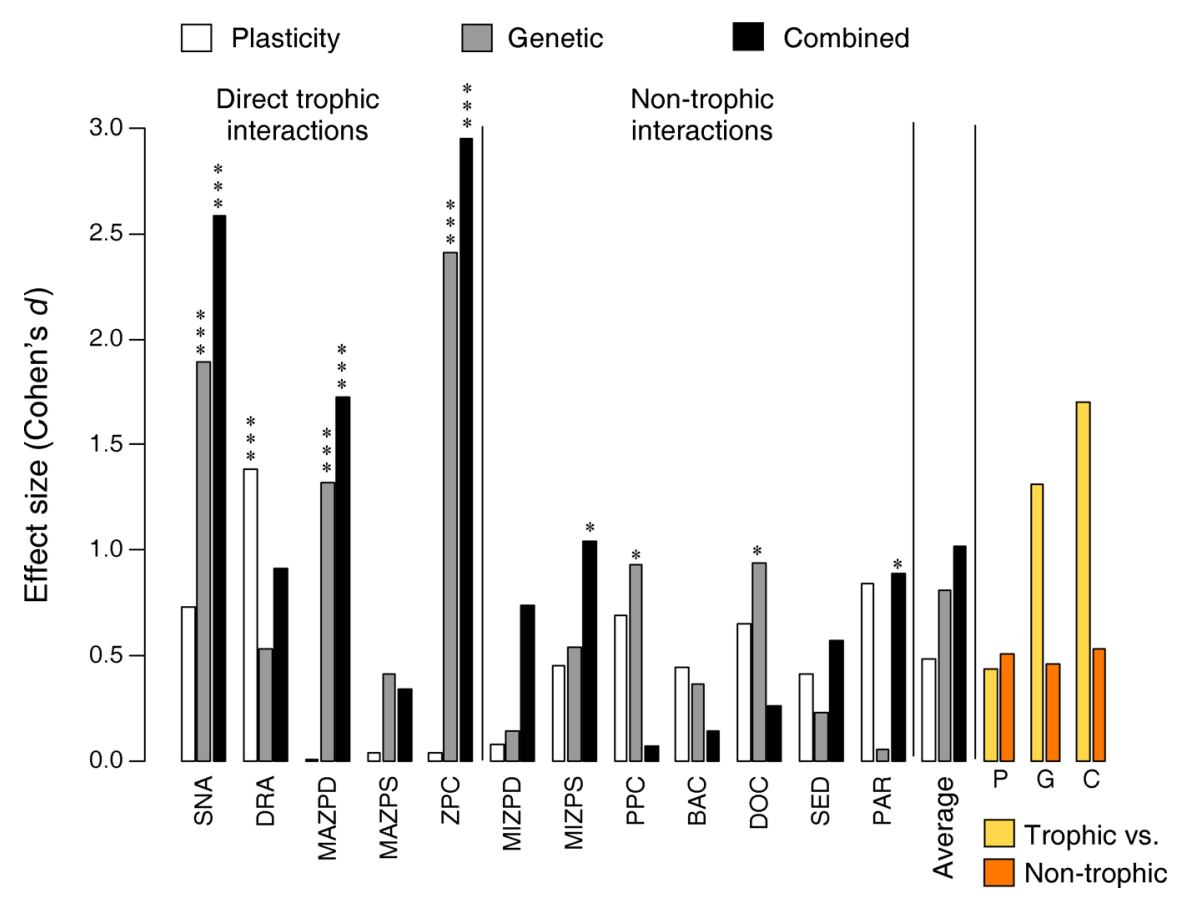

FIG. 5. Distribution of effect sizes for metrics presented in Table 1; MAZPS stands for macrozooplankton size, ZPC for macrozooplankton community, MIZPD for microzooplankton density, MIZPS for microzooplankton size, BAC for benthic algae cover, DOC for dissolved organic compounds, SED for sedimentation rate, and PAR for photosynthetically active radiation. Shown are effect sizes (Cohen's $d$ ) for the different contrasts (BB vs. BL for plasticity, shown in white; BL vs. LL for genetic effects, shown in gray; LL vs. BB for combined effects, shown in black) at the end of the experiment on the $y$-axis and the different ecosystem metrics on the $x$-axis. Ecosystem parameters are divided into trophic and non-trophic effects. Significant Cohen's $d$ values (based on randomization tests, Bailey et al. 2009) are presented as * $P<0.05$; ** $P<0.01$; *** $P<0.001$. On the right side of the graph, we report for each contrast the average of all effect sizes and effect size averages of trophic (yellow bars) and nontrophic (orange bars) metrics. Trophic and non-trophic effects are ordered from plasticity $(\mathrm{P})$, to genetic $(\mathrm{G})$ to combined $(\mathrm{C})$, from left to right.

other as opposed to a non-diversified population? We consider this an important and unanswered question that straddles the disciplinary divide between evolutionary biology and ecosystems ecology. Here, we present experimental evidence that divergent whitefish species that evolved within a geologically young adaptive radiation in a large pre-alpine European lake have contrasting effects on aquatic ecosystems in experimental mesocosms. Our experiments build on previous work showing that divergence between sympatric stickleback species (Harmon et al. 2009), but also differences among allopatric populations of guppy (Bassar et al. 2010) and alewife (Palkovacs and Post 2009) can affect ecosystem properties and functions. However, because these previous studies used wild-caught fish rather than fish reared in a common garden, our study is the first to separate ecosystem effects of closely related species that are due to heritable trait divergence and those due to trait differences arising from environmentally induced phenotypic plasticity. We found significant effects of both genetic species divergence and environmentally induced plastic divergence. Genetic effects were more frequent than plasticity effects, although some large effects were observed for both (Fig. 5). Overall, this suggests that the phenotypic and genetic divergence among species that builds up as a consequence of speciation and adaptive radiation within ecosystems is indeed the most likely explanation for contrasting ecosystem effects of such species, but phenotypic plasticity can modify some of the ecosystem effects of these species.

\section{Whitefish divergence affects ecosystems}

By the end of the experiment, our multivariate analysis of ecosystem divergence revealed significant differences between treatments, and this supports some aspects of our initial predictions (Fig. 1d, e). It supports our first prediction that ecosystem divergence results from both genetic divergence between whitefish species and from phenotypic plasticity within species (Fig. 3a, b, Appendix D). It also supports our third prediction, in that the contrast in ecosystem state was largest between the BB and LL treatments (Fig. 3a, b, Appendix D), but it does not support our second prediction of overall stronger genetic than plasticity effects. It supports our fourth prediction that ecosystem effects are stronger for trophic than for non-trophic interactions (Fig. 3b, c, Appendix D). Our analysis of the temporal dynamics of ecosystem metrics over the course of the experiment showed that our whitefish treatments had strongly 
contrasting effects on the abundances of their prey (Fig. $4 \mathrm{a}-\mathrm{c})$. To some extent, the direction of these effects can be explained by known differences in phenotypes, feeding efficiency, and ecology of whitefish (Fig. 2; Mookerji et al. 1998, Harrod et al. 2010, LundsgaardHansen et al. 2013, Roesch et al. 2013). Consistent with our previous work, which demonstrated genetically based species differences in foraging traits using the same individuals reared in a common garden (Lundsgaard-Hansen et al. 2013), the phenotypically benthic $C$. sp. "Bodenbalchen" reduced the abundance of benthic prey more strongly than the phenotypically limnetic $C$. zugensis, whereas the latter species reduced the abundance of limnetic prey more strongly. When raised on limnetic food, the benthic species became intermediate with respect to its effects on some metrics describing the prey community (Fig. 4). The strongly benthic prey Dreissena was similarly weakly affected by the benthic species raised on limnetic food and the limnetic species; but its abundance was strongly reduced by the benthic species raised on benthic food. This finding is also consistent with results from our previous work where we also found evidence for adaptive plasticity in the feeding efficiency on benthic food of the same pair of whitefish species (Fig. 2; Lundsgaard-Hansen et al. 2013).

In our experiment, whitefish also differentially affected properties of the ecosystem other than their prey, such as the dynamics of phytoplankton through time (Fig. 4d). Phytoplankton abundance in lake ecosystems is often regulated by organisms at higher trophic levels in both the pelagic and benthic food chain (Vanni and Findlay 1990). As a result, their dynamics can be influenced by how fish feed both on pelagic macrozooplankton and on benthic filter feeders (e.g., Dreissena). Phytoplankton biomass was negatively correlated with the size of macrozooplankton in the middle of the experiment and with the abundance of Dreissena at later dates. This suggests that understanding the temporal dynamics of contrasting ecosystem effects that result from trait differences among predators may depend on the life history, generation time, and food-web position of their prey.

In radiations of northern temperate fish, there are many species pairs with eco-morphological differentiation that is similar to that among our studied whitefish species pair (Schluter 2000), and we know next to nothing about how repeatable the effects of their evolutionary divergence on ecosystems are, in terms of quality and magnitude of effect. Future studies should test for repeatability of ecosystem effects by replicating experiments with independently evolved species pairs. Our results also suggest that the presence of diversity in predators may change temporal dynamics of predatorinduced ecosystem effects compared to systems with only one type of predator (for instance, the dynamics of phytoplankton in our mesocosms). However, such effects of ecological complementarity that have evolved in adaptive radiation need to be addressed explicitly in future experiments. Currently we know little about how effects observed in simplified and small model ecosystems (i.e., our study; Harmon et al. 2009, Palkovacs and Post 2009, Bassar et al. 2012) scale to effects in real ecosystems in nature. Thus, future studies would gain valuable additional insights if they were performed under natural or seminatural conditions.

\section{Distribution of effect sizes}

In our experiment, contrasting ecosystem effects of divergent whitefish were not randomly distributed in the ecosystem, and seemed to result more frequently from direct trophic interactions of whitefish with their prey than from indirect effects on lower trophic levels. This is consistent with dampening of top-down effects in trophic cascades (Micheli 1999, Dickie et al. 2012), but it may alternatively be explained by multiple indirect effects that act in opposing directions and result in weak overall net effects on lower trophic levels (Bassar et al. 2012).

We found large ecosystem effects resulting from both genetic divergence and phenotypic plasticity, but genetic effects were more common in our experiment. This is in agreement with a recent study showing that the genetically based divergence in the rates of population growth among Daphnia clones (reared in a common garden) alters consumer-resource dynamics and ecosystem function (Walsh et al. 2012). We cannot exclude that our genetic effects include some maternal effects, because we used first generation lab-bred individuals. However, there is strong evidence for heritability of several key ecological traits in whitefish (Gagnaire et al. 2013), and maternal effects on some of these, such as the number of gill rakers, are very unlikely.

\section{Summary and future directions}

Here, we studied species which are known to be genetically differentiated in ecologically relevant traits, i.e., feeding-related morphological traits and feeding behavior (Vonlanthen et al. 2012, Lundsgaard-Hansen et al. 2013), and found that they have strongly contrasting effects on their prey and other characteristics of the ecosystem. There is independent evidence that foraging traits can be under divergent natural selection in whitefish adaptive radiations (Rogers and Bernatchez 2007, Vonlanthen et al. 2009, Renaut et al. 2012, Gagnaire et al. 2013, Hebert et al. 2013, LundsgaardHansen et al. 2013), and that anthropogenic modification of the environment has caused contemporary evolutionary change in these fish through changes in the selection regimes (Bittner et al. 2010, Vonlanthen et al. 2012, Hudson et al. 2013). Some intriguing questions for future research would be to (1) identify which heritable traits underlie whitefish-mediated modifications to ecosystems, (2) test how variation in these traits affect ecosystems, (3) determine how such phenotypic effects compare to other agents of environmental change, and (4) test whether the whitefish-mediated 


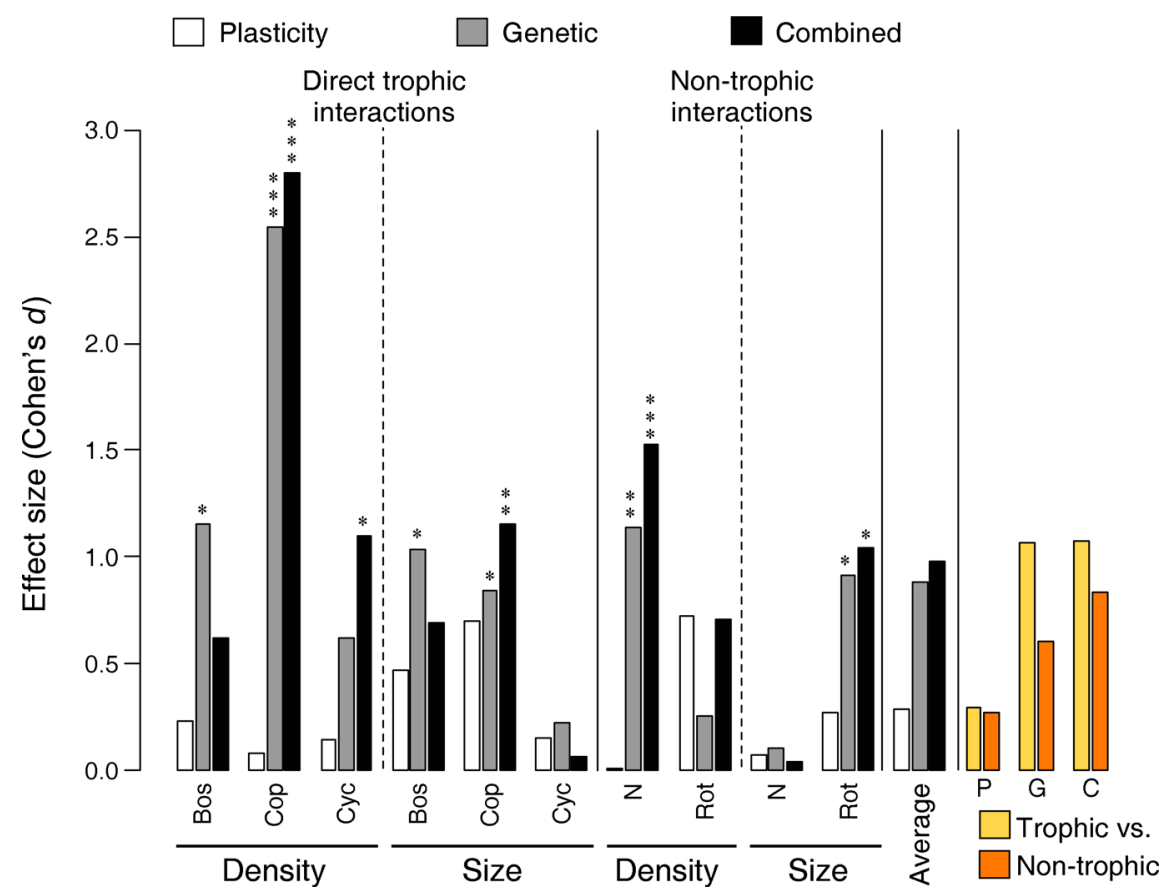

FIG. 6. Distribution of effect sizes for the different contrasts on zooplankton density and size per taxon; Bosmina (Bos), calanoid copeods (Cop), cylopoid copepods (Cyc), copepod nauplii $(\mathrm{N})$, and Rotifera (Rot). Shown are effect sizes (Cohen's $d$ ) for the different contrasts (as in Fig. 5) at the end of the experiment on the $y$-axis and the different metrics on the $x$-axis. Acarids, Daphnia, and Ostracoda were very rare (relative abundance at each date within each tank always $<1 \%$ ), and are thus not included here. Zooplankton taxa are divided into trophic and non-trophic interactions. On the right, averaged effect size per contrast over all metrics (including densities and sizes) as well as averages for trophic and non-trophic metrics are shown. Bar colors are as in Figs. 1 and 4. Significant randomization tests are indicated with $* P<0.05 ; * * P<0.01$; *** $P<0.001$.

environmental modifications affect selection pressures on subsequent whitefish generations and on other organisms. In addition, if plasticity in foraging traits, for example, significantly affects an ecosystem state that is preserved across generations, then feedbacks between plasticity and the environment could promote the evolution of traits with a genetic basis, including evolution of the reaction norm itself (Yamamichi et al. 2011). To investigate such ideas, future studies should measure the magnitude, dimensionality, and persistence of ecosystem effects of traits and trait diversity (Matthews et al. 2014), and quantify whether such modifications to the ecosystem alter environmental sources of natural selection (Yoder et al. 2010). Such work may require larger experimental ecosystems, but would help us understand whether the contrasting ecosystem effects of species that emerge from adaptive radiations tend to either promote or reduce ecological opportunity for further divergence, and whether such effects play an important role in the process of diversification itself (Erwin 2008, Losos 2010, Yoder et al. 2010, Matthews et al. 2014).

\section{ACKNOWLEDGMENTS}

We thank Sascha Di Piazza, Samuel Eberhardt, Johannes Hellmann, Doris Hohmann, Erwin Schäffer, Corinne Schmid, Daniel Steiner, and Andreas Taverna for support with the set- up of the experiment and data collection. Special thanks goes to Lucie Greuter for discussion and valuable comments on earlier versions of the manuscript. We acknowledge financial support by the EAWAG Action Field Grant Aqua Diverse-understanding and predicting changes in aquatic biodiversity.

\section{Literature Cited}

Agrawal, A. A. 2001. Phenotypic plasticity in the interactions and evolution of species. Science 294:321-326.

Bailey, J. K., et al. 2009. From genes to ecosystems: a synthesis of the effects of plant genetic factors across levels of organization. Philosophical Transactions of the Royal Society B 364:1607-1616.

Bassar, R. D., R. Ferriere, A. Lopez-Sepuldre, M. C. Marshall, J. Travis, C. M. Pringle, and D. N. Reznick. 2012. Direct and indirect ecosystem effects of evolutionary adaptation in the Trinidadian guppy (Poecilia reticulata). American Naturalist 180:167-185.

Bassar, R. D., et al. 2010. Local adaptation in Trinidadian guppies alters ecosystem processes. Proceedings of the National Academy of Sciences USA 107:3616-3621.

Baum, J. K., and B. Worm. 2009. Cascading top-down effects of changing oceanic predator abundances. Journal of Animal Ecology 78:699-714.

Bentzen, P., and J. D. McPhail. 1984. Ecology and evolution of sympatric sticklebacks (Gasterosteus): specialization for alternative trophic niches in the Enos Lake species pair. Canadian Journal of Zoology 62:2280-2286.

Bernatchez, L. 2004. Ecological theory of adaptive radiation: an empirical assessment from Coregonine fishes (Salmoniformes). Pages 175-207 in A. P. Hendry and S. C. Stearns, 
editors. Evolution illuminated. Oxford University Press, Oxford, UK.

Bittner, D., L. Excoffier, and C. R. Largiader. 2010. Patterns of morphological changes and hybridization between sympatric whitefish morphs between whitefish morphs (Coregonus spp.) in a Swiss lake: a role for eutrophication? Molecular Ecology 19:2152-2167.

Blank, N., A. G. Hudson, P. Vonlanthen, O. Seehausen, C. R. Hammerschmidt, and D. B. Senn. 2013. Speciation leads to divergent methylmercury accumulation in sympatric whitefish. Aquatic Sciences 75:261-273.

Borer, E. T., E. W. Seabloom, J. B. Shurin, K. E. Andersson, C. A. Blanchette, B. Broitman, S. D. Cooper, and B. S. Halpern. 2005. What determines the strength of a trophic cascade? Ecology 86:528-537.

Bouton, N., F. Witte, and J. J. M. van Alphen. 2002. Experimental evidence for adaptive phenotypic plasticity in a rock-dwelling cichlid fish from Lake Victoria. Biological Journal of the Linnean Society 77:185-192.

Burnham, K. P., and D. R. Anderson. 1998. Model selection and multimodel inference, a practical information-theoretic approach. Second edition. Springer, New York, New York, USA.

Cohen, J. 1988. Statistical power analysis for the behavioral sciences. Second edition. Lawrence Erlbaum, Hillsdale, New Jersey, USA.

Day, T., and J. D. McPhail. 1996. The effect of behavioural and morphological plasticity on foraging efficiency in the threespine stickleback (Gasterosteus sp.). Oecologia 108: 380-388.

Dickie, I. A., T. Fukami, J. P. Wilkie, R. B. Allen, and P. K. Buchanan. 2012. Do assembly history effects attenuate from species to ecosystem properties? A field test with woodinhabiting fungi. Ecology Letters 15:133-141.

Erwin, D. H. 2008. Macroevolution of ecosystem engineering, niche construction, and diversity. Trends in Ecology and Evolution 23:304-310

Gagnaire, P. A., E. Normandeau, S. A. Pavey, and L. Bernatchez. 2013. Mapping phenotypic, expression and transmission ratio distortion QTL using RAD markers in the lake whitefish (Coregonus clupeaformis). Molecular Ecology 22:3036-3048.

Grant, P. R. 1986. Ecology and evolution of Darwin's finches. Princeton University Press, Princeton, New Jersey, USA.

Hanski, I. 2012. Eco-evolutionary dynamics in a changing world. Annals of the New York Academy of Sciences 1246:117.

Harmon, L. J., B. Matthews, S. Des Roches, J. M. Chase, J. B. Shurin, and D. Schluter. 2009. Evolutionary diversification in stickleback affects ecosystem functioning. Nature 458:11671170 .

Harrod, C., J. Mallela, and K. Kahilainen. 2010. Phenotypeenvironment correlations in a putative whitefish adaptive radiation. Journal of Animal Ecology 79:1057-1068.

Hebert, F. O., S. Renaut, and L. Bernatchez. 2013. Targeted sequence capture and resequencing implies a predominant role of regulatory regions in the divergence of a sympatric lake whitefish species pair (Coregonus clupeaformis). Molecular Ecology 22(19):4896-4914.

Hudson, A. G., P. Vonlanthen, E. Bezault, and O. Seehausen. 2013. Genomic signatures of relaxed selection associated with speciation reversal in whitefish. BMC Evolutionary Biology 13:108.

Hudson, A. G., P. Vonlanthen, and O. Seehausen. 2011. Rapid parallel adaptive radiations from a single hybridogenic ancestral population. Proceedings of the Royal Society B 278:58-66.

Karvonen, A., B. Lundsgaard-Hansen, J. Jokkela, and O. Seehausen. 2012. Differentiation in parasitism among eco- types of whitefish segregating along depth gradients. Oikos 122:122-128

Legendre, P., and E. D. Gallagher. 2001. Ecologically meaningful transformations for ordination of species data. Oecologia 129:271-280.

Losos, J. B. 2010. Adaptive radiation, ecological opportunity and evolutionary determinism. American Naturalist 175: 623-639.

Losos, J. B., A. C. Douglas, D. Glossip, R. Goellner, A. Hampton, G. Roberts, N. Haskell, P. Taylor, and J. Ettling. 2000. Evolutionary implications of phenotypic plasticity in the hindlimb of the lizard Anolis sagrei. Evolution 54:301305.

Lu, G., and L. Bernatchez. 1999. Correlated trophic specialization and genetic divergence in sympatric lake whitefish ecotypes (Coregonus clupeaformis): support for the ecological speciation hypothesis. Evolution 53:1491-1505.

Lundsgaard-Hansen, B., B. Matthews, P. Vonlanthen, A. Taverna, and O. Seehausen. 2013. Adaptive plasticity and genetic divergence in feeding efficiency during parallel adaptive radiation of whitefish (Coregonus spp.). Journal of Evolutionary Biology 26:483-498.

Matthews, B., L. De Meester, C. G. Jones, B. W. Ibelings, T. J. Bouma, V. Nuutinen, J. van de Koppel, and J. Odling-Smee. 2014. Under niche construction: an operational bridge between ecology, evolution, and ecosystem science. Ecological Monographs 84:245-263.

Matthews, B., S. Hausch, C. Winter, C. A. Suttle, and J. B. Shurin. 2011a. Contrasting ecosystem effects of morphologically similar copepods. Plos ONE 6:e26700.

Matthews, B., et al. 2011b. Toward an integration of evolutionary biology and ecosystem science. Ecology Letters 14:690-701.

McQueen, D. J., M. R. S. Johannes, J. R. Post, T. J. Stewart, and D. R. S. Lean. 1989. Bottom-up and top-down impacts on freshwater pelagic community structure. Ecological Monographs 59:289-309.

Micheli, F. 1999. Eutrophication, fisheries and consumerresource dynamics in marine pelagic ecosystem. Science 285:1396-1398.

Miner, B. G., S. E. Sultan, S. G. Morgan, D. K. Padilla, and R. A. Relyea. 2005. Ecological consequences of phenotypic plasticity. Trends in Ecology and Evolution 20:685-692.

Mookerji, N., C. Heller, H. J. Meng, H. R. Bürgi, and R. Müller. 1998. Diel and seasonal patterns of food intake and prey selection by Coregonus sp. in re-oligotrophicated Lake Lucerne, Switzerland. Journal of Fish Biology 52:443-457.

Nosil, P. 2012. Ecological speciation. Oxford University Press, Oxford, UK.

Palkovacs, E. P., M. T. Kinnison, C. Correa, C. M. Dalton, and A. P. Hendry. 2012. Fates beyond traits: ecological consequences of human-induced trait change. Evolutionary Applications 5:183-191.

Palkovacs, E. P., and D. M. Post. 2009. Experimental evidence that phenotypic divergence in predators drives community divergence in prey. Ecology 90:300-305.

Pigliucci, M. 2005. Evolution of phenotypic plasticity: Where are we going now? Trends in Ecology and Evolution 20:481486.

Post, D. M., E. P. Palkovacs, E. G. Schielke, and S. I. Dodson. 2008. Intraspecific phenotypic variation in a predator affects community structure and cascading trophic interactions. Ecology 89:2019-2032.

R Development Core Team. 2010. R: A language and environment for Statistical Computing. Vienna, Austria. www.R-project.org

Renaut, S., N. Maillet, E. Normandeau, C. Sauvage, N. Derome, S. M. Rogers, and L. Bernatchez. 2012. Genomewide patterns of divergence during speciation: the lake 
whitefish case study. Philosophical Transactions of the Royal Society B 367:354-363.

Robinson, B. W., and K. J. Parsons. 2002. Changing times, spaces and faces: tests and implications of adaptive morphological plasticity in the fishes of northern postglacial lakes. Canadian Journal of Fisheries and Aquatic Sciences 59:1819-1833.

Roesch, C., B. Lundsgaard-Hansen, P. Vonlanthen, A. Taverna, and O. Seehausen. 2013. Experimental evidence for trait utility of gill raker number in adaptive radiation of a north temperate fish. Journal of Evolutionary Biology 26: $1578-1587$.

Rogers, S. M., and L. Bernatchez. 2007. The genetic architecture of ecological speciation and the association with signatures of selection in natural lake whitefish (Coregonus sp., Salmonidae) species pairs. Molecular Biology and Evolution 24:1423-1438.

Rundle, H. D., L. Nagel, J. W. Boughman, and D. Schluter. 2000. Natural selection and parallel speciation in sticklebacks. Science 287:306-308.

Schluter, D. 1995. Adaptive radiation in sticklebacks: trade-offs in feeding performance and growth. Ecology 76:82-90.

Schluter, D. 2000. The ecology of adaptive radiation. Oxford University Press, Oxford, UK.

Schoener, T. W. 2011. The newest synthesis: understanding the interplay of evolutionary and ecological dynamics. Science 331:426-429.

Seehausen, O. 2009. Speciation affects ecosystems. Nature 458: $1122-1123$.

Shurin, J. B., E. T. Borer, E. W. Seabloom, K. Anderson, C. A. Blanchette, B. Broitman, S. D. Cooper, and B. S. Halpern. 2002. A cross-ecosystem comparison of the strength of trophic cascades. Ecology Letters 5:785-791.

Tabachnik, B. G., and L. S. Fidell. 2006. Using multivariate statistics. Allyn and Bacon, Boston, Massachusetts, USA.

Teng, J., and K. S. McCann. 2004. Dynamics of compartmented and reticulate food webs in relation to energetic flows. American Naturalist 164:85-100.
Vanni, M. J., and D. L. Findlay. 1990. Trophic cascades and phytoplankton community structure. Ecology 71:921-937.

Vonlanthen, P., D. Bittner, A. G. Hudson, K. A. Young, R. Müller, B. Lundsgaard-Hansen, D. Roy, S. Di Piazza, C. R. Largiader, and O. Seehausen. 2012. Anthropogenic eutrophication drives extinction by speciation reversal in parallel adaptive radiations. Nature 482:357-363.

Vonlanthen, P., D. Roy, A. G. Hudson, C. R. Largiader, D. Bittner, and O. Seehausen. 2009. Divergence along a steep ecological gradient in lake whitefish (Coregonus sp.). Journal of Evolutionary Biology 22:498-514.

Walsh, M. R., J. P. De Long, T. C. Hanley, and D. M. Post. 2012. A cascade of evolutionary change alters consumerresource dynamics and ecosystem function. Proceedings of the Royal Society B 279:3184-3192.

Walsh, M. R., and D. M. Post. 2011. Interpopulation variation in a fish predator drives evolutionary divergence in prey in lakes. Philosophical Transactions of the Royal Society B 278: 2628-2637.

West-Eberhard, M. J. 1989. Phenotypic plasticity and the origins of diversity. Annual Review of Ecology, Evolution, and Systematics 20:249-278.

West-Eberhard, M. J. 2003. Developmental plasticity and evolution. Oxford University Press, Oxford, UK.

Woods, P. J., R. Müller, and O. Seehausen. 2009. Intergenomic epistasis causes asynchronous hatch times in whitefish hybrids, but only when parental ecotypes differ. Journal of Evolutionary Biology 22:2305-2319.

Wund, M. A., J. A. Baker, B. Clancy, J. L. Golub, and S. A. Foster. 2008. A test of the flexible stem model of evolution: ancestral plasticity, genetic accommodation, and morphological divergence in the threespine stickleback radiation. American Naturalist 172:449-462.

Yamamichi, M., T. Yoshida, and A. Sasaki. 2011. Comparing the effects of rapid evolution and phenotypic plasticity on predator-prey dynamics. American Naturalist 178:287-304.

Yoder, J. B., et al. 2010. Ecological opportunity and the origin of adaptive radiations. Journal of Evolutionary Biology 23: $1581-1596$.

\section{Supplemental Material}

\section{Appendix A}

Sampling overview (Ecological Archives E095-238-A1).

\section{Appendix B}

Measuring ecosystem variables (Ecological Archives E095-238-A2).

Appendix C

PCA results summary (Ecological Archives E095-238-A3).

Appendix D

Between treatments Kruskal-Wallis ANOVA and post-hoc $t$ tests (Ecological Archives E095-238-A4).

\section{Appendix E}

Community composition of macrozooplankton at the end of the experiment (Ecological Archives E095-238-A5).

\section{Appendix F}

Backward model selection phytoplankton (Ecological Archives E095-238-A6). 